\title{
FLOWS ON SOLVMANIFOLDS
}

\author{
BY L. AUSLANDER AND L. GREEN ${ }^{1}$ \\ Communicated by Lawrence Markus, July 2, 1963
}

Let $G$ be a connected, simply connected solvable Lie group and let $C$ be a closed subgroup such that $G / C$ is compact. Further, let $g(t)$ be a one parameter subgroup of $G$. Then $g(t)$ induces, acting to the left on $G / C$, a one parameter group of transformations. We will call $G / C$ a representation of a compact solvmanifold and the flow generated by $g(t)$ a $G$-induced flow. In [1] and [2] results concerning special $G$-induced flows were discussed in detail or research announced. It is the purpose of this note to state one aspect of the problem of $G$-induced flows and outline the solution that is now available. Full details and discussions will be presented elsewhere.

Let $G / C$ be a representation of a compact solvmanifold. Our main problem may be stated as follows: Give a necessary and sufficient condition for the existence of an ergodic $G$-induced flow on $G / C$. We will now outline our solution to this problem.

Algebraic preliminaries. Let $L(G)$ denote a solvable Lie algebra which may be taken over the real or complex fields. Then $L(G)$ is said to be of Type $(R)$ if all the roots of the algebra are either 0 or pure imaginary. One of the main algebraic facts we will need is the following:

THEOREM. Given a solvable Lie algebra $L(G)$ there exists a unique minimal ideal $H$ such that $L(G) / H$ is Type $(R)$.

We will also need the companion concept of a really regular element of $L(G) . X \in L(G)$ will be called really regular if it is

(1) regular,

(2) ad $(X)$ has a maximal number of eigenvalues with nontrivial real part. The set of really regular elements is dense in $L(G)$.

THEOREM. $G / C$ has a G-induced ergodic flow if and only if there is a really regular element $X$ of $L(G)$ such that $\exp (t X)$ induces an ergodic flow.

REDUCTION TheOREM. A really regular element in $L(G)$ induces an ergodic flow on $G / C$ if and only if it induces an ergodic flow on $\mathrm{Cl}(G / C H)$, where $H$ is the unique minimal analytic normal subgroup of $G$ such that $G / H$ is Type $(R)$ and $\mathrm{Cl}$ denotes the closure operation.

${ }^{1}$ Supported by NSF research contracts. 
Hence we see that we have reduced the problem to the Type $(R)$ case.

More algebra I. Let $S$ be a Type $(R)$ solvable group, which is connected and simply connected. Then there exists a unique nilpotent analytic group $N$ and a compact abelian group $T$ of automorphisms of $N$ such that

(a) $S \subset T \cdot N$.

(b) Let $p: S \rightarrow N$ be the projection mapping.

Then $p$ is a homeomorphism of $S$ onto $N$.

(c) $S$ and $N$ generate $T \cdot N$.

We will call $T \cdot N$ the minimal splitting of $S$.

More algebra II. Let $C$ be a closed subgroup of a connected, simply connected Type $(R)$ solvable group $S$. Let $T \cdot N$ be a minimal splitting for $S$. Then $S / N \cap C$ is compact and $C / N \cap C$ is a finite group. Further, the projection mapping $p: S \rightarrow N$ induces a homeomorphism of $S / N \cap C$ onto $N / N \cap C$.

Definition. We will say that a one-parameter group in $S$ is in general position if its projection onto $T$ is dense in $T$, where $T \cdot N$ is the minimal splitting of $S$. Then one can easily see that the image of a really regular element of $G$ is in general position in $S$ and conversely, given an element of $S$ in general position there exist really regular elements of $G$ which project onto it.

Second Reduction Theorem. Let $S=G / H$, where $H$ is the unique minimal analytic normal subgroup of $G$ such that $G / H$ is Type $(R)$. $A G$-induced flow on $S / C$ is ergodic if and only if there exists an $S$ induced flow which is ergodic on $S / N \cap C$.

Theorem. Let $S$ be of Type $(R)$. There exists an S-induced ergodic flow on $S / N \cap C$ if and only if the null space of $(t-I)$, for all $t \in T$, contains a vector in general position in $N / C_{0}[N, N]$ relative to the lattice $C / C_{0}([N, N] \cap C)$, where $C_{0}$ is the identity component of $C$.

\section{REFERENCES}

1. L. Auslander, L. Green and F. Hahn, Flows on homogeneous spaces, Annals of Mathematics Studies No. 53, Princeton Univ. Press, Princeton, N. J., 1963.

2. L. Auslander and F. Hahn, Discrete transformations on tori and flows on solvmanifolds, Bull. Amer. Math. Soc. 68 (1962), 614-615.

PuRdue University and

UNIVERSITY OF MiNNESOTA 